\title{
BMJ Open Meditation involving people with cancer, medical staff and witnesses: a pilot study exploring improvement in wellness and connectedness
}

\author{
V Prevost (D) , ${ }^{1,2}$ Bénédicte Clarisse, ${ }^{2}$ Alexandra Leconte, ${ }^{2}$ Claire Delorme, ${ }^{2}$ \\ Sandrine Benoit, ${ }^{2}$ Titi Tran $^{2}$
}

To cite: Prevost V, Clarisse B, Leconte $\mathrm{A}$, et al. Meditation involving people with cancer, medical staff and witnesses: a pilot study exploring improvement in wellness and connectedness. BMJ Open 2021;11:e048164. doi:10.1136/ bmjopen-2020-048164

- Prepublication history for this paper is available online. To view these files, please visit the journal online (http://dx.doi. org/10.1136/bmjopen-2020048164).

Received 21 December 2020 Accepted 20 October 2021

Check for updates

(c) Author(s) (or their employer(s)) 2021. Re-use permitted under CC BY-NC. No commercial re-use. See rights and permissions. Published by BMJ.

${ }^{1}$ Normandie Université, UNICAEN, INSERM U1086 ANTICIPE, Caen, France

${ }^{2}$ Centre François Baclesse Centre de Lutte Contre le Cancer, Caen, France

Correspondence to Dr V Prevost;

virginie.prevost@unicaen.fr

\section{ABSTRACT}

Introduction Mindfulness meditation is likely to promote better management of stress, pain and negative emotions. We propose to address the benefit of meditation in an open setting associating people with cancer (target population), medical staff and witnesses (neither patient nor medical staff). This study aims (1) to evaluate the effects of meditation on wellness improvement and (2) to identify criteria and modalities for a subsequent randomised study.

Methods and analysis We propose a longitudinal pilot study consisting of a non-randomised experimental preintervention/ postintervention survey. The intervention consists in delivering a meditation programme (12 weekly meditation sessions of 1.5 hours each), specifically adapted to our target population and addressing our research hypothesis in an open setting involving people with cancer, medical staff and witnesses (equally distributed in two groups of 15 participants). The main objective is to evaluate participants' adherence to the programme. The effects of meditation will be evaluated on stress, quality of life, feeling of personal effectiveness, on the development of mindfulness and empathy, and on satisfaction and perception of a change in quality of life. We will also measure the putative added value of 'meditating together'. This study is expected to allow validating the evaluation tools and refining the modalities of the workshops. We expect to demonstrate the evolution that this meditation-based intervention induces in the participants. We aim to promote bridge-building, between patients, medical staff but also others. In this way, one's own suffering may be understood in the light of others' suffering, thereby promoting the sense of otherness and giving insights into 'living better with'. This exploratory study will investigate the relevance of this hypothesis, which could then be explored by a randomised study.

Ethics and dissemination The protocol was approved by the local ethics committee (Comité de Protection des Personnes Est II). Trial findings will be published in peer-reviewed journals.

Trial registration number NCT04410185.

\section{INTRODUCTION}

\section{Context}

The pain of patients, the suffering of medical staff... Quality of life remains impaired in $44 \%$ of patients 5 years after cancer diagnosis. ${ }^{1}$
Strengths and limitations of this study

- This pilot study is the first step in a comprehensive project aiming to evaluate the added value of meditation in an open setting addressing people with cancer, medical oncology staff and witnesses.

- This pilot study aims to evaluate participants' adherence to the mindfulness meditation programme in an open setting.

- Outcome measures will include assessment of quality of life, stress in participants, disease management in patients, as well as evaluation of mindfulness qualities, effects of 'meditating together' and participant satisfaction.

- Small sample size and pilot design are limitations of this study.

The findings from this study will lay the foundations for the design and setting of an appropriate randomised study.

More than one in two patients undergoing cancer treatment experiences pain. ${ }^{2}$ Medical staffs also suffer and are increasingly threatened by burn-out, particularly in oncology. ${ }^{3}$ The simplistic view would be that there are medical staff who help, on the one hand, and those who are helped, on the other. In fact, they reflect each other: each member of the medical staff is home to a wounded person and each patient to an inner healer. ${ }^{4}$ Vulnerability, which is inherent in the human condition, is not reserved only for those who experience illness, ${ }^{5}$ and suffering is shared, whether one is a medical professional, a patient or a third party who is witness to the suffering. In this state of interdependence where care is a shared function, ${ }^{5}$ the bond between patient and medical professional becomes a therapeutic relationship. As De Hennezel puts it: 'Relief and transformation of anxiety are only possible when the medical staff and the cared-for can meet, humbly, on the same ground of human experience, 
recognizing that we all carry within us joy and pain, life and death, and that it is through the things that hurt and penetrate us that we become vulnerable, therefore open to others and truly human' (our translation). ${ }^{6}$ The encounter between patient and medical professional may thus be understood as fertile ground for more creative, collaborative and interactive healthcare.

\section{A way to meet the challenge: mindfulness meditation}

In response to the pain of patients as well as the suffering of medical staff, integrative medicine, with its emphasis on complementary therapeutic approaches, the holistic consideration of subjects and their autonomy, ${ }^{7}$ is a relevant approach. Within the complementary alternative medicines (CAM), mindfulness meditation (MM) meets these criteria and constitutes a global approach likely to improve health and allow better management of stress, pain and negative emotions. Mindfulness, as defined by Kabat-Zinn in 1979 , is a natural state of consciousness that we can learn to develop through meditation exercises that require us to intentionally focus on the present moment without judging the experience that is unfolding moment by moment. ${ }^{8}$ MM, adapted from the age-old Buddhist meditation practices, is thus part of the contemporary world and is proposed today as 'lay meditation'. Kabat-Zinn proposed it in the form of a protocolised programme accessible to all, called mindfulness-based stress reduction (MBSR). ${ }^{8}$ The concept of mindfulness is now integrated into the field of positive psychology. ${ }^{9}$

\section{The pain demonstrated beneficial effects in patients, in medical} staff...

In oncology, the analysis of systematic reviews on nonpharmacological interventions demonstrated an improvement in quality of life through meditation. ${ }^{1011}$ Zhang et $a l$ 's recent synthesis of 14 studies, involving 1505 people with breast cancer, highlighted the significant benefits of an MBSR programme on emotional well-being, anxiety, stress and depression. ${ }^{12}$ However, the data in the literature are heterogeneous so studies are difficult to compare. Notable issues concern the modalities of interventions (choice of protocol, duration, intensity, etc) and their evaluation (size of the population, control group, etc) ${ }^{13}$ A recent study on CAM in oncology found little scientific evidence for MM due to the lack of studies on the subject. ${ }^{14}$ Two recent studies underlined the importance of taking into account and understanding the parameters that may explain the occurrence of unpleasant experiences such as fear and anxiety that are potentially induced by meditation. ${ }^{15} 16$

Regarding the evaluation of the impact of meditation on caregiver suffering, Dobkin et al showed that CAM has a positive impact on clinicians' well-being by reducing stress and burn-out and making them more present, which in turn affects the quality of communication with their patients, who feel better understood. ${ }^{17}$ Recent metaanalyses have shown that meditation has proven to reduce work distress and improve caregiver well-being, ${ }^{18}$ and to reduce caregiver stress significantly. ${ }^{19}$

\section{Research hypothesis}

And if meditating together created an added benefit?

As a response to suffering, meditation can, through a process of inner transformation, improve our well-being in the sense of our own psyche and strengthen our bonds. It offers the opportunity to build a bridge between the medical professional (who sometimes needs care) and the person being cared for (who wishes to play an active role in his or her illness), with whom the witness can be associated, in solidarity, with the suffering he or she may be confronted with. Bridge-building between professional and patient is a powerful way to establish mutual trust, whereby the professional abandons his/her position as an expert and the patient takes on the role of the one who is being treated. This makes it possible to generate a contractual relationship built on a partnership that promotes patient autonomy ${ }^{20}$ and the humanisation of care. Thus, 'care does not belong to a caste of medical staff... It is a shared and creative function of medical staff and patients who, together, create a unique dynamic, particularly due to the specific nature of the subjects they are... Care and subjects are inseparable' (our translation). ${ }^{5}$ Yet could we not go one step further? In addition to establishing a link between patients and medical staff and in order to break out of the context of 'illness', would it not be possible to include 'non-patient' and 'non-care-giving' subjects, that is, witnesses, in meditation workshops with a view to destigmatising the patient as a patient and the hospital as a place of care, in a context where the diversity of the group is an asset and enriches exchange and learning?

\section{Aim of the study}

The objective is to offer MM in collective workshops involving health professionals, patients, their families and friends or any person wishing to participate. Our hypothesis is that having the patient, the caregiver and the witness occupy the same space is likely to modify their respective representations and postures in order to promote a more favourable health path for our target audience, that is, people with cancer. It is only human nature to focus on one's own suffering: the patient on his/her physical and/or mental pain, the doctor on his/her professional workload, etc. Opening the sessions up simultaneously to patients, medical staff and witnesses might lead the participants to look at each other in a different light, at what can be shared, and at how some answers might be found regarding the issue of 'living better with'.

By creating collective benefit for our three populations, meditating together might promote a sense of openness to each other and the feeling of sharing each other's existence. Being together makes people more permeable to each other and allows them to grasp who the others really are, even if each person in the triad retains his or her own specificities. The research hypothesis is therefore that beyond the individual benefit that it can bring 


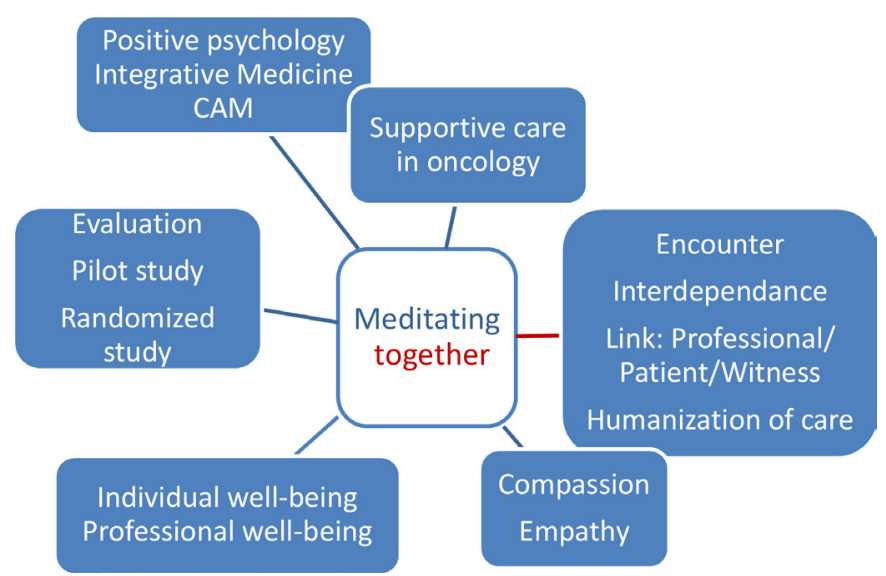

Figure 1 Cluster of arguments in favour of 'meditating together' hypothesis. CAM, complementary alternative medicines.

to participants, particularly in the daily management of stress, meditating together can provide additional benefit in terms of well-being and strengthened links between patients, medical staff and witnesses (figure 1).

Although the effects of meditation are increasingly documented, few hospitals currently offer it in their supportive care and its potential benefits remain undervalued. Our project implements meditation in hospital and evaluates its feasibility and benefits through a pilot study. The question then arises as to whom it should be offered. Some studies directly target the population concerned, that is, patients. Others focus on the benefits of meditation among medical staff, the rationale being that the quality of care they provide will be better if their own well-being is improved. ${ }^{17}$

Our hypothesis is that meditating together could be beneficial. Although supportive care has previously been offered occasionally to medical staff and patients in joint workshops in a clinical context (without evaluation research), the idea that an additional benefit could be obtained by conducting the experience outside the context of the hospital and the illness alone has not been explored. If patients, medical staff and witnesses meditate together, it might lead to the emergence of another type of deeper link that engenders more empathy, more mutual understanding, and a greater sense of collective belonging among the participants.

This project, therefore, functions on two levels. In clinical terms, there is a need to offer patients global care that is complementary to the care they receive in an open, 'decompartmentalised' setting. In terms of research, the effectiveness of the approach needs to be validated to enable its dissemination. At the clinical level, patients tend not to be considered as unique and whole beings, so the care they receive does not encompass their psychosocial, spiritual and community dimensions in addition to their biological and physical dimensions. MM is opening up the field of care. It constitutes a form of mental training, a 'way of being', so that patients can live better with their disease. It can also offer solutions to the psychological suffering and lifestyle-related stress than is widespread in society today. Yet a recent publication ${ }^{14}$ found that CAM, including MM, is still insufficiently evaluated in people with cancer, so the scientific evidence supporting it is sparse, thus limiting the opportunity to recommend and disseminate it.

\section{An innovative pilot study}

To our knowledge, there is currently no study addressing (1) the benefit of meditation in an open setting associating patients, medical staff and witnesses, or (2) the putative added value of such an approach, particularly for patients, compared with that of an approach specifically addressing each type of population. Yet there is a strong demand from patients for CAM and MM, a secular technique whose benefits have been validated scientifically. ${ }^{10-12}$

This pilot study is the first step in a comprehensive project aiming to evaluate the added value of 'meditating together'. Experiments are being conducted at the Stress Clinic created in Massachusetts by Kabat-Zinn, who was the first to introduce secular meditation into medicine, where patients and medical staff come together to meditate, ${ }^{4}$ although the added benefit of meditating together has not yet been evaluated. The initial step is to validate the relevance of our hypothesis through an exploratory phase focusing on our three populations. Indeed, before studying the putative benefits of MM, we need to test whether our participants consider it feasible and acceptable. For example, will medical staff consider it relevant and feasible to meditate together with patients and vice versa? The pilot study described herein seeks to resolve these preliminary issues.

\section{METHODS AND ANALYSIS The meditation programme}

A teacher who is an expert in meditation will be responsible for designing the programme and setting up the workshops. As a qualified instructor of MBSR programmes and a member of the Medit-Ageing Research Group, she has taught meditation in the European Silver Santé Study project piloted by INSERM in Caen, France. This project seeks to identify the factors of well-being and mental health in seniors. ${ }^{21}$ Within that study, she developed a programme based on mindfulness and empathy that is adapted to our hypothesis concerning the benefits of 'meditating together'. In addition to the qualities of attentiveness and non-judgement, the programme will also focus on peace of mind, caring and compassion.

Regarding the organisation of the meditation workshops, the reference protocol for stress reduction based on MM is the MBSR protocol, ${ }^{8}$ which is frequently used in practice and has already been evaluated. ${ }^{12}$ This 8 -week programme includes 8 weekly 2.5-hour meditation sessions and an 8-hour retreat day. However, a duration of 2.5 hours per session would not have been suitable for all people with cancer, who are our central population. For our project, the workshops will, therefore, be offered in a format with more but shorter sessions over a period of 3 months. It will thus include 12 sessions of 1.5 hours 


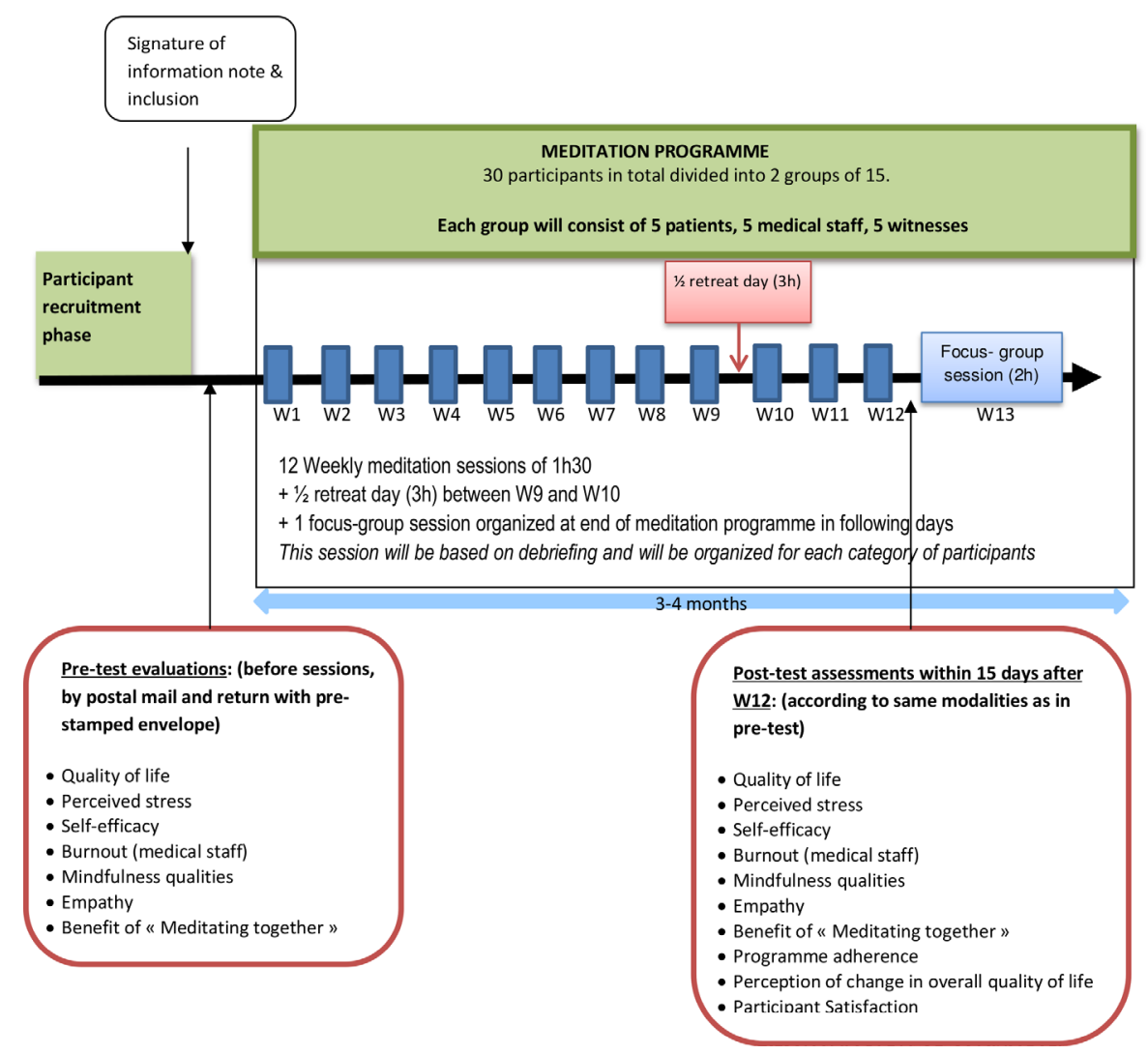

Figure 2 Study design.

of meditation per week and a half-day of 3 hours. Two groups of 15 participants equally distributed among our three populations (30 participants in total) will be established (figure 2).

As part of the programme, participants will be required to complete daily practice exercises and meditation sessions at home. To guide them, written and audio materials specially designed for this purpose by the teacher will also be provided. Finally, individual follow-up will be offered, if necessary, by phone, videocall or face-toface discussion in order to resolve any problems raised during the sessions ${ }^{15} 16$ that cannot be managed within the group. The meditation sessions and the deepening session (retreat) will take place outside the hospital, allowing for meetings and exchanges in a neutral, friendly and convivial environment (Pôle des Formations et de Recherche en Santé of Caen University), a pleasant setting conducive to exchange that is close to the Comprehensive Cancer Centre François Baclesse.

\section{Selection of participants}

The main parameter for inclusion is curiosity about meditation and motivation to be part of a study on it. Those recruited should have no current or previous experience of regular or intensive meditation or comparable practice. For people with cancer, their health status must allow them to attend the sessions. Concerning the medical staff, any medical and/or paramedical staff at the Centre François Baclesse involved in the management of people with cancer (doctors, nurses, nursing assistants, radiotherapy/ radiology operatives) will be eligible. As for witnesses, any person not belonging to the above-mentioned categories will be eligible. They can be relatives of the patient or non-medical staff at the François Baclesse Centre. They can also be people from outside the centre to the extent that they are not health professional workers.

The eligibility of subjects presenting significant vulnerability such as very advanced stage of cancer disease with life-threatening consequences, alcohol or drug dependence, severe depression, severe social anxiety, recent bereavement, etc. will be assessed on a case-by-case basis. In this regard, the participant's degree of commitment to the project will be a determining criterion. All participants will provide written informed consent.

\section{Study methodology}

This is a longitudinal monocentre pilot study consisting of a non-randomised experimental preintervention/postintervention survey with minimal risks and constraints. The intervention under consideration here is the delivery of a meditation programme in an open setting, involving patients, medical staff and witnesses (the latter also constituting a control group). As per instructions for protocols papers, the study is ongoing and not carried out at the time of submission of this article (18 December 2020).

\section{Objectives of pilot study}

The main objective is to test the feasibility of our working hypothesis regarding the potential benefit of proposing meditation in an open setting. The main criterion is, 
therefore, to evaluate the participants' adherence to the 13-session meditation programme. In drug treatment studies, good adherence is commonly defined as a $80 \%$ or more ratio of doses taken out of prescribed doses. Similarly, in our study, participants will be considered adherent if they attend at least $80 \%$ of the 13 sessions of the whole meditation programme. The secondary objective will be to assess the putative effects of meditation on stress and quality of life, on feelings of personal effectiveness, and on the development of mindfulness and empathy. In addition, we will evaluate the putative added value of offering workshops common to our three populations, especially for the target population of patients. In medical staff, we will specifically evaluate the degree of burnout. The satisfaction of all participants with the MM programme will be measured, as well as the perception of a change in their overall quality of life.

From a methodological point of view, we intend (1) to validate the tools chosen and their relevance with regard to these evaluations and (2) to refine the eligibility criteria in the workshops. Rather than seek to demonstrate the effectiveness of MM, we aim to document the evolution that $\mathrm{MM}$ induces in the participants from preintervention to postintervention.

\section{Tools for evaluation}

The following tools will be used to assess the impact of MM on the participants. They will be administered as shown in figure 2 .

\section{Assessment of quality of life, stress in participants and disease} management in patients

Quality of life will be assessed in a global manner using a visual analogue scale, a tool that has proven its value in this field. ${ }^{22}$ Another objective is stress reduction. The Perceived Stressed Scale is a 10 -item questionnaire validated in French, ${ }^{23}$ adapted from Cohen et $a .^{24}$ It is a simple and quickly administered tool to measure (preintervention and postintervention) the extent to which life situations are generally perceived as threatening, that is, unpredictable, uncontrollable and painful. Participants' perception of a change in their overall quality of life will be measured at the end of the programme. $^{25}$

In the caregiver population, burn-out will be assessed before and after the programme with the Maslach Burnout Inventory ${ }^{26}$ translated by Alain and Gévry. ${ }^{27}$ This scale explores the feeling of emotional exhaustion, dehumanisation and personal fulfilment at work.

Since one of the objectives of MM is to optimise adaptation skills, we believe it is relevant to evaluate the ability and motivation to cope with difficult situations. The Generalised Self Efficacy Scale ${ }^{28}$ validated in French, ${ }^{29}$ which is commonly used in positive psychology, will be used for this purpose before and after the programme. This scale, which is appropriate outside the specific context of the illness, will be administered to all participants.

\section{Evaluation of the qualities of mindfulness}

We have developed a questionnaire that focuses on participants' perceptions of meditation, that is, true and false ideas about it. Various tools can be used to assess the qualities of mindfulness. ${ }^{30}$ The questionnaire we will use is the Freiburg Mindfulness Inventory, ${ }^{31}$ a self-report tool developed in 2001 for assessing mindfulness in everyday life. Its short 14-item version ${ }^{32}$ has been validated in French. ${ }^{33}$

The interpersonal reactivity index developed by Davis ${ }^{34}$ in 1980 and validated in French ${ }^{35}$ will be used to evaluate the spontaneous tendency to empathy. The Jefferson Scale of Empathy ${ }^{36}$ will also be used to explore the participants' perception of the importance of empathy in care. This scale, which evaluates empathy in the health field, was first developed for health professionals ${ }^{36}$ and then adapted for health students. ${ }^{37}$ In the latter, the items are written in a neutral manner, which will allow the validated French version ${ }^{38}$ to be used by all our participants.

\section{Evaluation of the putative benefit of 'meditating together'}

While the evaluation of the putative benefit of 'meditating together' seems relevant to the integrative approach in clinical psychology, it is not developed in clinical practice and therefore remains unevaluated. Insofar as the proposed project postulates that 'meditating together' could provide added value to our target population, we propose to use questionnaires specifically constructed in the framework of this study to shed light on our problem. Different aspects of this issue will be evaluated using Likert scales to allow us to refine the overall response, depending on the status of the participant (patient, medical staff, witness). They will focus on participants' feelings about meditating together in terms of discomfort, value and facilitation of communication and compassion between patients/medical staff/ witnesses.

To further explore the putative added value of 'meditating together' and with a view to designing a relevant randomised study, we propose a complementary qualitative approach. For this purpose, at the end of the meditation programme, the participants' opinion on the proposal to 'meditate together' will be collected during a 2-hour session using the focus-group method. ${ }^{39}$ Thanks to the dynamics of the group, we will be able to validate and clarify the formulation of the main hypotheses of our project, and even to encourage the emergence of relevant issues raised by the participants. The external evaluator in charge of data collection will launch the questions to be discussed, the main subject of which will be the main hypothesis of this pilot study, namely the putative added value of 'meditating together'. It will also be an opportunity to compare the opinions of the participants on the practical aspects of the programme (organisation of the sessions, etc), its content, its effects and its avenues for improvement. This will provide information complementary that obtained by the closed questions in the satisfaction questionnaire. 


\section{Evaluation of participant satisfaction}

At the end of the programme, the satisfaction of the participants will be assessed by measuring different dimensions: the schedule of the programme, its content and the pedagogical techniques used, the quality of the relationship with the trainer and the exchange with/ among the participants.

\section{Statistical overview}

The main criterion is the adherence of participants to the whole programme.

No power calculation was performed to determine the sample size. The pilot study will be conducted with 30 participants, in 2 groups of 15 participants each: 5 patients $/ 5$ caregivers $/ 5$ controls. Statistical analysis will be mainly descriptive, based on both quantitative and qualitative methods.

First, adherence to the meditation programme will be estimated with the proportion of participants who will have realised the 13 sessions, with the $95 \%$ CI. The assessment of the impact of the meditating practice will be based on the quantitative scores from above mentioned questionnaires. Assessable population will include participants who will have performed at least one session. Score statistics will be calculated before and after the meditating programme. The qualitative analysis will consist to identify some potential obstacles to meditation, to better understand the reasons of a possible failure of the meditating intervention in order to redesign an adapted modality of intervention for meditation practice.

\section{Patient and public involvement}

The research question emerged from informal discussions between a researcher and a meditation teacher. The latter, in addition to the sessions' conduction is involved in research (design and construction of an adapted meditation programme, choice of evaluation tools, etc).

Focus group interviews will allow to accurately assess and integrate participant's group experiences to refinement of our research hypothesis. Participants will not be involved in the recruitment and conduct of the study. Results from this study will be disseminated to each participant as a lay summary of the findings and through results presentation at Comprehensive Cancer Centre François Baclesse.

\section{DISCUSSION}

\section{Expected outcomes and prospects}

This pilot project aims to improve well-being, to strengthen the links between medical staff, patients and witnesses, and to raise awareness about how to live better together by proposing a shared activity in an open setting. The expected benefits, therefore, primarily concern the relief of psychological suffering. Enabling better understanding between carers and cared-for and taking a different look at oneself and at others are experiences which, beyond the scope of the project, aim to promote exchange and interaction between those concerned. For people with cancer, pain and other symptoms related to the disease and its treatments might be better managed. In addition, the pain experienced by medical staff, which is mainly due to professional overload and the difficulty of being in daily contact with the disease, will be considered. Its management through meditation will have a beneficial impact on the way medical staff are able to interact with their patients.

Developing a relationship of trust that takes the specificity of each person into account and supporting the patient in a holistic manner are both challenges that, if overcome, can avoid the dehumanisation of care. The collective benefit expected from this study is to develop our interdependence and our humanity and to live better together by recognising suffering as a shared characteristic, irrespective of our specific problems experienced as patient, professional or witness.

If this study leads to satisfactory results in terms of participation, adherence of participants to the MM programme and benefits related to meditation, it will help to clarify the main judgement criterion that would be used for a randomised study aiming at answering the research hypothesis and demonstrating the effectiveness of open-ended CAM. The programme limitations identified during this exploratory phase will help improve the protocol for subsequent phases. Thus, the criteria and modalities for recruiting participants into the programme as well as the meditation programme itself and its components (content, length of sessions, frequency, follow-up, etc) and the questionnaires used could be optimised.

This pilot study will, therefore, lay the foundations for the construction of an appropriate randomised study that meets the needs of patients. This exploratory study will allow us to identify the most relevant parameters and to refine our research hypothesis in order to answer it through a rigorous evaluation conducted with a robust methodology.

\section{Study status}

This study has received ethical approval from the local ethics committee (Est II Comity of Person Protection, Besançon, France) on 25 May 2020. The participant recruitment phase lasted from 1 June 2020 to 9 September 2020. The meditation programme began on 10 September 2020 and ended in late February 2021. Data analysis is ongoing.

\section{Ethics and dissemination}

The study protocol was approved by the local ethics committee (Comité de Protection des Personnes Est II, Ref CNRIPH protocol: 20.03.20.55708). The approved protocol version is V.1.2 dated from 18 May 2020. Trial findings will be published in peer-reviewed journals.

Acknowledgements The Northwest Data Centre (CTD-CNO, supported by grants from the French National League Against Cancer (LNC) and the French National Cancer Institute (INCa)) is acknowledged for managing the data. Management and the Communication Service of the Comprehensive Cancer Centre François Baclesse are acknowledged for their support and confidence in carrying out their project. Special thanks go to Sandrine Lasalle-Polinière, Director of Human Resources, Sandrine Benoit, Director of Care, and Dr Agnès Palix, Occupational Physician. They warmly thank the participants (patients, medical staff and witnesses), who 
will agree to be involved in the study. VP and TT thank Jean-Gérard Bloch for the inspiring scope of the University Diploma (DU) Medicine, Meditation, Neurosciences. VP thanks Patrick Emo for the stimulating discussions about the project and for 'introducing' her to Cynthia Fleury. We also acknowledge Ray Cooke for copyediting the manuscript.

Contributors VP, BC, AL and TT wrote the manuscript and devised the study concept and design. $B C$ and $A L$ were responsible for overseeing the methodological section. All authors (VP, BC, AL, CD, SB and TT) were involved in drafting the manuscript or revising it critically for important intellectual content. VP and BC supervised the entire work. All authors have given final approval of the version to be published. Each author has participated sufficiently in the work to take public responsibility for appropriate portions of the content.

Funding This study was supported by grants from the Fondation de France (Appel à projets " Soigner, Soulager, Accompagner: projets de recherche » 2019. No Engagement 00101605) and by the French Cancéropôle Nord-Ouest (Appel à projets Emergence 2020).

Disclaimer The funding agencies were not involved in the design or conduct of the study, nor in the collection, management, analysis, or interpretation of the data. They were not involved in drafting the manuscript.

\section{Competing interests None declared.}

Patient and public involvement Patients and/or the public were involved in the design, or conduct, or reporting, or dissemination plans of this research. Refer to the Methods section for further details.

Patient consent for publication Not applicable.

Provenance and peer review Not commissioned; externally peer reviewed.

Open access This is an open access article distributed in accordance with the Creative Commons Attribution Non Commercial (CC BY-NC 4.0) license, which permits others to distribute, remix, adapt, build upon this work non-commercially, and license their derivative works on different terms, provided the original work is properly cited, appropriate credit is given, any changes made indicated, and the use is non-commercial. See: http://creativecommons.org/licenses/by-nc/4.0/.

\section{ORCID iD}

V Prevost http://orcid.org/0000-0003-2873-6063

\section{REFERENCES}

1 Institut National du CAncer. VICAN5, 2018. Available: https://www. e-cancer.fr/Expertises-et-publications/Le-point-sur/La-vie-cinq-ansapres-un-diagnostic-de-cancer [Accessed Dec 2020].

2 van den Beuken-van Everdingen MHJ, Hochstenbach LMJ, Joosten EAJ, et al. Update on prevalence of pain in patients with cancer: systematic review and meta-analysis. J Pain Symptom Manage 2016;51:1070-90.

3 Yates M, Samuel V. Burnout in oncologists and associated factors: a systematic literature review and meta-analysis. Eur $J$ Cancer Care 2019;28:e13094.

4 Santorelli S. Guéris-toi toi-même : ce que la méditation apporte la médecine. Paris: Editions des Arènes, 2017.

5 Fleury C. Le soin est un humanisme. Tracts Gallimard $N^{\circ} 6$. Paris: Editions Gallimard, 2019.

6 De Hennezel M, Gutton P. Et si vieillir libérait la tendresse... Collection Old'up : inventer sa vieillesse. Paris, 2019.

$7 \mathrm{NCCIH}$. Complementary, alternative, or integrative health: What's name? Available: https://www.nccih.nih.gov/health/ integrativehealth [Accessed Dec 2020].

8 Kabat-Zinn J. Mindfulness-based interventions in context: past, present, and future. Clin Psychol Sci Pract 2003;10:144-56.

9 Bernard M, Zimmermann G, Favez N. Which place for positive psychology in psychotherapy? theoretical and empirical perspectives. Prat psychol 2011;17:301-13.

10 Duncan M, Moschopoulou E, Herrington E, et al. Review of systematic reviews of non-pharmacological interventions to improve quality of life in cancer survivors. BMJ Open 2017;7:e015860.

11 Xunlin NG, Lau Y, Klainin-Yobas P. The effectiveness of mindfulnessbased interventions among cancer patients and survivors: a systematic review and meta-analysis. Support Care Cancer 2020;28:1563-78.

12 Zhang Q, Zhao H, Zheng Y. Effectiveness of mindfulness-based stress reduction (MBSR) on symptom variables and health-related quality of life in breast cancer patients-a systematic review and meta-analysis. Support Care Cancer 2019;27:771-81.

13 Carlson LE, Zelinski E, Toivonen K, et al. Mind-Body therapies in cancer: what is the latest evidence? Curr Oncol Rep 2017;19:67.

14 Bosacki C, Vallard A, Gras M, et al. [Complementary and alternative medicines in cancer patients]. Bull Cancer 2019;106:479-91.

15 Baer R, Crane C, Miller E, et al. Doing no harm in mindfulness-based programs: conceptual issues and empirical findings. Clin Psychol Rev 2019;71:101-14.

16 Schlosser M, Sparby T, Vörös S, et al. Unpleasant meditation-related experiences in regular meditators: prevalence, predictors, and conceptual considerations. PLoS One 2019;14:e0216643.

17 Dobkin PL, Bernardi NF, Bagnis Cl. Enhancing clinicians' well-being and patient-centered care through mindfulness. J Contin Educ Health Prof 2016;36:11-16.

18 Spinelli C, Wisener M, Khoury B. Mindfulness training for healthcare professionals and trainees: a meta-analysis of randomized controlled trials. J Psychosom Res 2019;120:29-38.

19 Burton A, Burgess C, Dean S, et al. How effective are MindfulnessBased interventions for reducing stress among healthcare professionals? A systematic review and meta-analysis. Stress Health 2017;33:3-13.

20 Tribonnière dela $X$. Être en relation avec Le patient. In: Pratiquer I'éducation thérapeutique. Chapitre: Elsevier Masson SAS, 2016: 4. 35-42.

21 Poisnel G, Arenaza-Urquijo E, Collette F, et al. The Age-Well randomized controlled trial of the Medit-Ageing European project: effect of meditation or foreign language training on brain and mental health in older adults. Alzheimers Dement 2018;4:714-23.

22 Conroy T, Guillemin F, Kaminsky MC. [Measure of quality of life in patients with metastatic colorectal cancer: techniques and main results]. Rev Med Interne 2002;23:703-16.

23 edQuintard B. Du stress objectif au stress perçu. In: Introduction la psychologie de la santé. M. Bruchon-Schweitzer et R. Dantzer. Paris: PUF, 1994: 43-66.

24 Cohen S, Kamarck T, Mermelstein R. A global measure of perceived stress. J Health Soc Behav 1983;24:385-96.

25 Fischer Det al. Capturing the Patient's View of Change as a Clinical Outcome Measure. JAMA 1999;282:1157-62.

26 Maslach C, Jackson SE. The measurement of experienced burnout. J Organ Behav 1981;2:99-113.

27 Alain \& Gévry. Maslach Burnout Inventory. In: Traduction Française. Palo Alto CA: Consulting Psychologist Press, 1986.

28 Schwarzer R, Jerusalem M. Generalized Self-Efficacy scale. In: Weinman J, Wright S, Johnston M, eds. Measures in health psychology: A user's portfolio. Causal and control beliefs. Windsor, England: NFER-NELSON, 1995: 35-7.

29 Dumont M, Schwarzer R, Jerusalem M. French Adaptation of the General Self-Efficacy Scale - Auto-efficacité Généralisée, Berlin, Germany 2000.

30 Trousselard M, Steiler D, Claverie D. How to assess mindfulness? Problems and future]. Encephale 2016;42:99-104.

31 Buchheld N, Grossman P, Walach H. Measuring mindfulness in insight meditation (vipassana) and meditation-based psychotherapy: the development of the Freiburg mindfulness inventory (FMI). J Medit Medit Res 2001;1:5-23.

32 Walach $\mathrm{H}$, Buchheld N, Buttenmüller V, et al. Measuring mindfulness - the Freiburg mindfulness inventory (FMI). Pers Individ Dif 2006;40:1543-55.

33 Trousselard M, Steiler D, Raphel C. Validation of a French version of the Freiburg mindfulness inventory (FMI) - short version: relationships between mindfulness and stress in an adult population. Bio Psycho Soc Med 2010;4:1-8.

34 Davis $\mathrm{MH}$. A multidimensional approach to individual differences in empathy. Available: https://www.uv.es/ friasnav/Davis_1980.pdf [Accessed Dec 2020].

35 Gilet A-L, Mella N, Studer J, et al. Assessing dispositional empathy in adults: a French validation of the interpersonal reactivity index (IRI). Can J Behav Sci 2013;45:42-8.

36 Hojat M, Mangione S, Nasca TJ, et al. The Jefferson scale of physician empathy: development and preliminary psychometric data. Educ Psychol Meas 2001;61:349-65.

37 Hojat M, Gonnella JS, Nasca TJ, et al. Physician empathy: definition, components, measurement, and relationship to gender and specialty. Am J Psychiatry 2002;159:1563-9.

38 Zenasni F, Boujut E, Bluffel du Vaure C. Development of a French language version of the Jefferson scale of physician empathy and association with practice characteristics and burnout in a sample of general practitioners. Int J Pers Cent Med 2012;2:759-66.

39 Kitzinger J. Qualitative research. Introducing focus groups. BMJ 1995;311:299-302. 